\title{
Borexino: recent solar and terrestrial neutrino results
}

\author{
Werner Maneschg* \\ Max-Planck-Institut für Kernphysik, Saupfercheckweg 1, D-69117 Heidelberg \\ E-mail: werner.maneschg@mpi-hd.mpg.de
}

\section{on behalf of the Borexino Collaboration:}

M. Agostini, S. Appel, G. Bellini, J. Benziger, D. Bick, G. Bonfini, D. Bravo, B. Caccianiga, F. Calaprice, A. Caminata, P. Cavalcante, A. Chepurnov, D. D’Angelo, S. Davini, A. Derbin, L. Di Noto, I. Drachnev, A. Etenko, K. Fomenko, D. Franco, F. Gabriele, C. Galbiati, C. Ghiano, M. Giammarchi, M. Goeger-Neff, A. Goretti, M. Gromov, C. Hagner, E. Hungerford, Aldo Ianni, Andrea Ianni, K. Jedrzejczak, M. Kaiser, V. Kobychev, D. Korablev, G. Korga, D. Kryn,

M. Laubenstein, B. Lehnert, E. Litvinovich, F. Lombardi, P. Lombardi, L. Ludhova,

G. Lukyanchenko, I. Machulin, S. Manecki, W. Maneschg, S. Marcocci, E. Meroni, M. Meyer, L. Miramonti, M. Misiaszek, M. Montuschi, P. Mosteiro, V. Muratova, B. Neumair, L. Oberauer, M. Obolensky, F. Ortica, M. Pallavicini, L. Papp, L. Perasso, A. Pocar, G. Ranucci, A. Razeto, A. Re, A. Romani, R. Roncin, N. Rossi, S. Schönert, D. Semenov, H. Simgen, M. Skorokhvatov, O. Smirnov, A. Sotnikov, S. Sukhotin, Y. Suvorov, R. Tartaglia, G. Testera, J. Thurn, M. Toropova, E. Unzhakov, A. Vishneva, R.B. Vogelaar, F. von Feilitzsch, H. Wang, S. Weinz, J. Winter, M. Wojcik, M. Wurm, Z. Yokley, O. Zaimidoroga, S. Zavatarelli, K. Zuber, and G. Zuzel

\begin{abstract}
Borexino is a multi-ton organic liquid scintillator detector at the underground-based Laboratori Nazionali del Gran Sasso, Italy. Due to the unprecedented ultra-low background levels and a stable detector operation for more than eight years, Borexino has succeeded to perform real-time spectroscopy of low energy solar neutrinos and of terrestrial antineutrinos. For the most recently detected pp neutrinos, which constitute $90 \%$ of the total neutrino flux from the Sun, a rate of $(144 \pm 13$ (stat) \pm 10 (syst) $) \cdot(100 \text { ton } \cdot d)^{-1}$ was found. This is consistent with the expectations of the Standard Solar Model and the LMA-MSW solution of the neutrino oscillation paradigm. Lately Borexino improved also its previous results on terrestrial antineutrinos by considering a higher exposure of almost $1 \mathrm{kton} \cdot \mathrm{yr}$. The measurement counted $23.7_{-5.7}^{+6.5}(\mathrm{stat})_{-0.6}^{+0.9}$ (syst) events, which rejects a non-observation of terrestrial antineutrinos at $5.9 \sigma$ C.L. In addition, the ratio of the radiogenic heat elements $T h$ and $U$ deduced from this measurement was found to be consistent with that of chondrite meteorites, which reflect the primordial composition of the Earth.
\end{abstract}

The European Physical Society Conference on High Energy Physics

22-29 July 2015

Vienna, Austria

${ }^{*}$ Speaker. 


\section{Introduction}

Borexino is a large scale organic liquid scintillator detector used mainly for the detection of solar neutrinos, but also of (anti)neutrinos from other extraterrestrial, terrestrial and artificial sources [1]. It is located deep underground at the Laboratori Nazionali del Gran Sasso, in central Italy. The overburden of $1.4 \mathrm{~km}$ thick dolomite calcareous rock reduces the cosmic muon flux by six orders of magnitude resulting in $\sim 3 \mathrm{~m}^{-2} \mathrm{~h}^{-1}$ [2]. The outermost part of Borexino is a water Cherenkov detector able to detect efficiently the residual muon flux and to attenuate the neutron and gamma radiation from the surrounding rock. Inside the water tank a stainless steel sphere (SSS) of $13.7 \mathrm{~m}$ diameter is embedded. It contains three shells with organic liquids which are separated by two thin transparent nylon vessels. The two outermost shells are filled with a non scintillating buffer (PC+DMP) which shields against radiation from the outer detector parts. The innermost vessel has a diameter of $8.5 \mathrm{~m}$ and contains 278 ton of a liquid scintillator solution (PC+PPO). Under assumption of secular equilibrium the scintillator has ultra-low $U$ and Th mass fractions of $\sim 10^{-17} \mathrm{~g} / \mathrm{g}$. Energy depositions within this radiopure scintillator produce scintillation light, which is collected by 2212 photomultiplier tubes mounted on the SSS and facing towards the center. An effective light yield of $\sim 500$ photoelectrons per deposited $\mathrm{MeV}$ has been obtained [3]. This allows to perform spectroscopy down to $0.05 \mathrm{MeV}$ with a good energy resolution which scales with $5 \% / \sqrt{E[\mathrm{MeV}]}$. The light generation, propagation and detection is fast, which allows to reconstruct the light emission vertex of an event from the time-of-flight of the detected photons belonging to it. The position, time and energy information of the events is a powerful data analysis ingredient that permits to define subvolumes of interest ('fiducial volume' cuts) as well as to search for coincident signal or background events. Moreover, a closer look at the signal time structure allows to introduce pulse shape discrimination methods able to distinguish, for instance, beta-like events from alpha particles.

Neutrinos interact with the molecules of the Borexino scintillator via elastic scattering on electrons $\left(v_{x}+e^{-} \rightarrow v_{x}+e^{-}\right)$. On the contrary, antineutrinos are identified via a coincident signal based on inverse beta decays $\left(\bar{v}_{x}+p \rightarrow n+e^{+}\right)$. The two detection channels do not interfere each other, which means that e.g. a temporary measurement with an artificial antineutrino source will not affect the ongoing solar neutrino program.

The simultaneous fulfillment of the three criteria, namely a scintillator with an extreme low background, a low energy threshold and the real-time information about position and energy of each single event, is unique and responsible for the large number of outstanding results obtained with the Borexino detector since start of operation in May 2007. The following chapters will focus on the latest results about solar neutrinos and terrestrial antineutrinos obtained with this detector.

\section{Solar neutrinos}

According to the Standard Solar Model (SSM), the electromagnetic radiation released from the Sun is generated by nuclear reactions in which ionized hydrogen atoms fuse into helium nuclei. The main process ensemble, in which $99 \%$ of the energy is produced, is the proton-proton chain. Electron neutrinos of characteristic energies accompaign the intermediate fusion processes. Their measurement can be used to test the SSM predictions as well as neutrino properties originating 
from neutrino oscillation scenarios. Among the different solar neutrino flux components, in the years from 2007 to 2010 (Phase I) Borexino succeeded to measure precisely the monochromatic ${ }^{7} \mathrm{Be}$ neutrinos $(0.86 \mathrm{MeV})$ [4]. Due to the achieved high radiopurity of the scintillator it was also possible to determine the ${ }^{8} \mathrm{~B}$ neutrino $(<14.6 \mathrm{MeV})$ rate with a low energy threshold of $3 \mathrm{MeV}$ [5] and to observe the monoenergetic pep neutrinos (1.44 MeV) [6].

However, background events mainly from ${ }^{85} \mathrm{Kr},{ }^{210} \mathrm{Bi}$ and ${ }^{14} \mathrm{C}$ decays contributing to the very low energy part of the spectrum still prevented the first direct detection of the pp neutrinos $(<0.42 \mathrm{MeV})$, which constitute $90 \%$ of the solar neutrino flux. In order to overcome the difficulties of such a fundamental measurement, a two-fold strategy has been pursued by the collaboration. In a first step extensive scintillator purification campaigns have been carried out in 2010-2011. These were based on water extractions and sparing of the scintillator with nitrogen of low argon and krypton content. The six purification cycles reduced the ${ }^{85} \mathrm{Kr}$ rate to a level consistent with zero [7], the ${ }^{210} \mathrm{Bi}$ rate by a factor of $\sim 3$, and the $U$ and Th concentrations at least by factor of $\sim 15$ and $\sim 5$, respectively. In a second step one had to deal with the remaining ${ }^{14} \mathrm{C}$ background $[8] .{ }^{14} \mathrm{C}$ is intrinsically present in the molecules of the hydro-carbon rich scintillator and thus cannot be removed by any purification method. Even though the Borexino scintillator was made from old mineral oil and the ${ }^{14} \mathrm{C} /{ }^{12} \mathrm{C}$ ratio has an extremely small value of $\approx 2.7 \times 10^{-18}$, the ${ }^{14} \mathrm{C}$ beta spectrum $(<0.16 \mathrm{MeV})$ broadened by the energy resolution largely dominates the spectral region up to $\sim 0.22 \mathrm{MeV}$. Due to the high trigger rate, ${ }^{14} \mathrm{C}$ events can also overlap with each other or with other background species and generate so-called pile ups. Their summed energy can easily exceed the recoil energy of the pp neutrinos $(<0.26 \mathrm{MeV})$. Thus, all these ${ }^{14} \mathrm{C}$ induced background contributions need to be independently characterized prior performing a final fit. By solving trigger threshold effects and leaving pile-up events aside, it was possible to fit the pure ${ }^{14} \mathrm{C}$ beta spectrum and to determine the rate as $(3.46 \pm 0.09) \times 10^{6} \cdot(100 \text { ton } \cdot d)^{-1}$. For the description of the ${ }^{14} \mathrm{C}$ pile-ups, a data-driven procedure was developed: real triggered physics events are overlapped artificially with other uncorrelated physics events leading to 'synthetic' pile-ups. These pile-ups, which do not suffer of e.g. position reconstruction effects, are then reconstructed using the same software and selection criteria applied on real non-superimposed data. The pile-up rate deduced via this procedure is $(321 \pm 7) \cdot(100 \text { ton } \cdot d)^{-1}$. In the following fits the ${ }^{14} \mathrm{C}$ beta and pile-up spectra and rates were fixed, allowing only for a variation consistent with their measured uncertainty.

For the first direct detection of the pp neutrinos with Borexino, a data set which covers the initial period of Phase II going from January 2012 to May 2013 (408 d live time) was used. To increase the signal-to-background ratio a fiducial volume was defined that keeps only events reconstructed within a truncated sphere of radius $3.021 \mathrm{~m}$ and $|\mathrm{z}|<1.67 \mathrm{~m}$. This cut includes 75.5 ton of scintillator, leading to a total fiducial exposure of $\approx 85$ ton $\cdot y r$. As depicted in Figure 1 the fit was performed in the $(165,590) \mathrm{keV}$ region. It was varied several times with slightly different conditions to test the robustness of the analysis and deduce potential systematic effects. The pp neutrino rate was determined to be [8]:

$$
R_{p p}=(144 \pm 13(\text { stat }) \pm 10(\text { syst })) \cdot(100 \text { ton } \cdot \mathrm{d})^{-1}
$$

This result allowed of several interpretations. By combining the rate with the knowledge about the neutrino oscillation properties as well as the neutrino cross sections and electron number density of the target, it was possible to deduce the solar pp neutrino flux of $(6.6 \pm 0.7) \times 10^{10} \mathrm{~cm}^{-2} \mathrm{~s}^{-1}$ at 


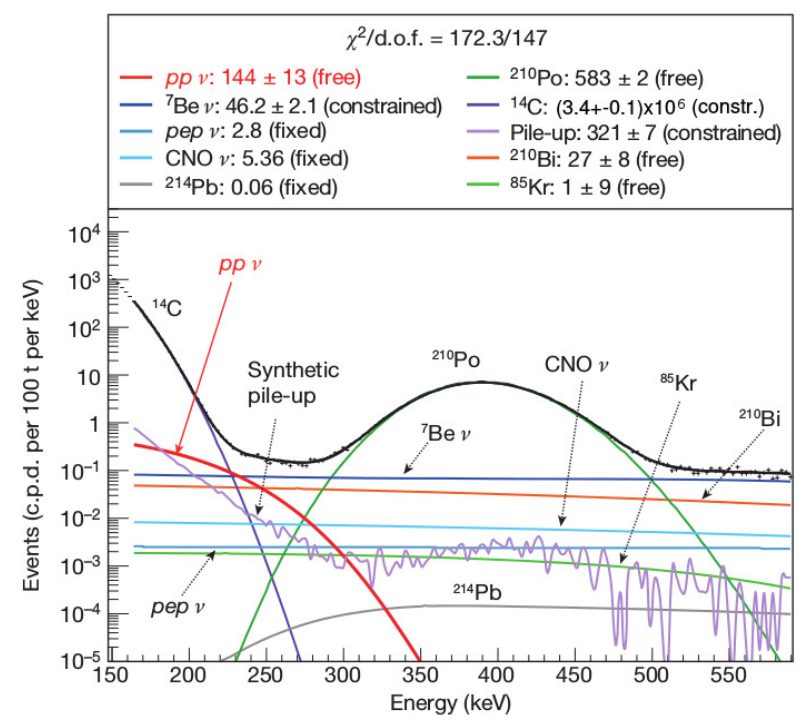

Figure 1: Detection of solar pp neutrinos with Borexino: best fit of the energy spectrum in the range $(165,590) \mathrm{keV}$. The neutrino and background rates are given in units of $(100 \mathrm{ton} \cdot \mathrm{d})^{-1}$.

one astronomical unit distance from the Sun. This is in well agreement with the SSM predictions. Vice versa, by comparing the measured rate with the one predicted from the SSM, the survival probability of electron neutrinos from the solar pp reactions of non being converted into other neutrino species, was found to be $0.64 \pm 0.12$. This is consistent with the most favored neutrino oscillation solution, in which flavor changes due to matter effects in the Sun are enhanced only above energies of several MeV (MSW-LMA). Finally, it turns out that the photon luminosity of the Sun, which is directly correlated with the neutrino luminosity within the SSM, is in agreement with the photon luminosity deduced from photospheric measurements. Since neutrinos produced in the solar core can escape the Sun within few seconds, while photons take several 10,000 years for it [9], the result proves that the Sun has shone stable over the mentioned period and is - against some hypotheses [10] - not responsible for terrestrial glacial epochs on such time scales.

\section{Terrestrial antineutrinos}

According to the Bulk Silicate Earth (BSE) model, one of the most popular descriptions of the Earth formation, composition and evolution, 19 out of 31-44 TW of thermal heat released from the surface is attributed to radioactive decays of mainly $\mathrm{U}$, Th, and $\mathrm{K}$ in the planetary crust and mantle. Next to the radiogenic heat, the decaying radioisotopes emit also antineutrinos with characterstic energy distributions. The expected terrestrial antineutrino flux is in the range of $10^{6} \mathrm{~cm}^{-2} \mathrm{~s}^{-1}$ only. This corresponds to a flux, which is five orders of magnitude less intense than that of solar neutrinos. Even though the flux is so small, two circumstances have allowed the detection of terrestrial antineutrinos with Borexino. First, antineutrinos with energies above $1.8 \mathrm{MeV}$, i.e. from $\mathrm{U}(<3.3 \mathrm{MeV})$ and $\mathrm{Th}(<2.3 \mathrm{MeV})$, but not from $\mathrm{K}(<1.3 \mathrm{MeV})$, can induce inverse beta decays (IBD) (see Section 1). The IBD process is followed by a coincident signal: A prompt signal is obtained from the positron annihilation. A delayed signal $(\tau \approx 255 \mu \mathrm{s}$ ) emerges from the subsequent 
neutron capture on hydrogen, which emits characteristic $2.2 \mathrm{MeV}$ gamma-rays. Both signals are strongly correlated in space and time and allow to reject efficiently background events. Second, the expected flux of antineutrinos from nuclear power plants is only factor of two higher than that of terrestrial origin. The reason is that Italy does not operate nuclear reactors, and other European stations are more distant from LNGS.

After two previous publications Borexino recently published a new result about terrestrial antineutrinos, which is based on a larger data set covering the period from December 2007 to March 2015 (2056 d live time) [11]. For an efficient reduction of muons and short-lived muonic by-products time cuts were applied which introduced a $10.4 \%$ dead time. For the reduction of the external radiation a time-variable fiducial volume cut was defined, which keeps only events reconstructed $>30 \mathrm{~cm}$ away from the non completely static nylon vessel. On average, $\approx 225$ ton of scintillator were considered. After applying the IBD and other cuts, a set of 77 antineutrino candidates remained, which is polluted by $1 \%$ of background events only. The prompt energy spectrum was fitted considering the expected spectral shape of terrestrial $U$, Th and reactor antineutrinos, and the residual background events (Figure 2). For the final exposure of (904 \pm 44$)$ (ton·yr) the following numbers of events were obtained [11]:

$$
\begin{aligned}
& N_{\text {terr }}=23.7_{-5.7}^{+6.5}(\text { stat })_{-0.6}^{+0.9}(\text { syst }) \\
& N_{\text {reac }}=52.7_{-7.7}^{+8.5}(\text { stat })_{-0.9}^{+0.7}(\text { syst })
\end{aligned}
$$

The uppermost result corresponds to a $5.9 \sigma$ observation of the terrestrial antineutrino signal. In addition, by treating the $\mathrm{U}$ and $\mathrm{Th}$ spectral contributions as free fit parameters, it was possible to separate the two flux contributions. The resulting $\mathrm{Th} / \mathrm{U}$ ratio is in agreement with the value 3.9, which was deduced from chondrites. These meteorites are thought to have conserved primordial material of which the Earth is also made of. From precise surveys of the local geological area [12] and global crust studies [13] it was possible to confine the radiogenic heat contribution from the local and global crust, and subsequently to find evidence of a signal originating from the mantle. The null hypothesis of such a positive signal was rejected at $98 \%$ confidence level.

\section{Summary and outlook}

Due to the unprecedented background levels achieved with the Borexino detector, it was possible to measure the solar ${ }^{7} \mathrm{Be},{ }^{8} \mathrm{~B}$, pep and pp neutrinos from the pp chain in real-time. Currently the collaboration aims at detecting the solar neutrinos from the catalytic CNO cycle. This process contributes to the solar energy output with $\sim 1 \%$ only, but plays a crucial role in astrophysical models. Moreover, such a neutrino measurement could help solving the more recently appeared discrepancies between SSM predictions and helioseimological data ('solar metallicity puzzle'). Terrestrial antineutrinos were also detected with high significance including a disentanglement of the two main contributions from $\mathrm{U}$ and $\mathrm{Th}$. However, more statistics will be needed to discriminate among different submodels of the Earth structure and its composition. Beyond this, the Borexino collaboration searches for other exotic rare processes such as Pauli forbidden transitions [14], solar axions [15] and the non-conservation of electric charge [16]. To conclude, within the SOX project 


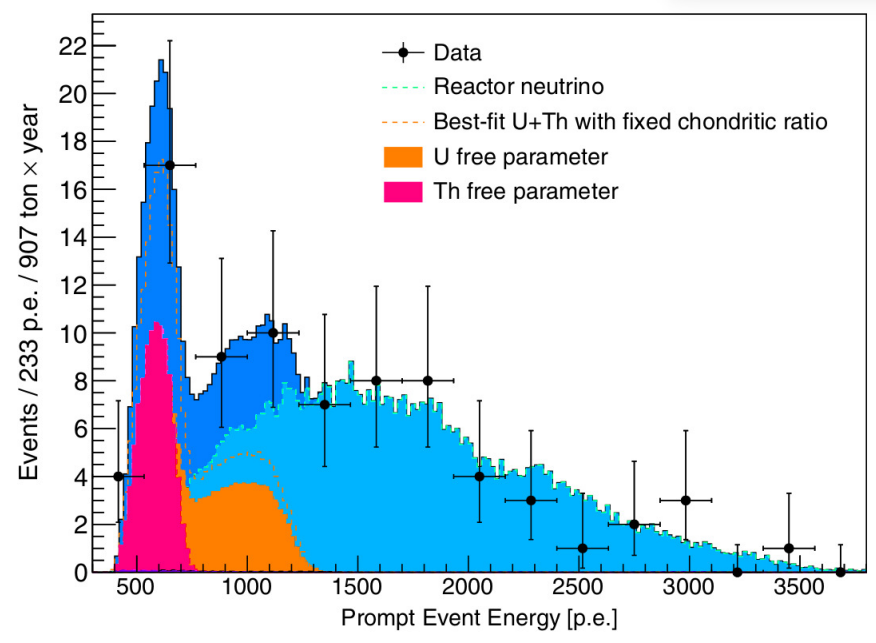

Figure 2: Borexino prompt light yield spectrum (units: photoelelectrons p.e.) of antineutrino candidates, including the terrestrial contribution from $U$ and Th decays. The best fit with the U/Th ratio (not) fixed is also shown.

[17] measurements with artificial antineutrino and neutrino sources are prepared for the search of a still undetected fourth, but sterile neutrino species.

\section{References}

[1] G. Alimonti et al. (Borexino Collaboration), Nucl. Inst. Meth. A 600 (2009) 568-593

[2] G. Bellini et al. (Borexino Collaboration), J. Cosm. Astrop. Phys. 05 (2012) 015

[3] H. Back et al. (Borexino Collaboration), J. Instr. 7 (2012) P10018

[4] G. Bellini et al. (Borexino Collaboration), Phys. Rev. Lett. 107 (2011) 141302

[5] G. Bellini at al. (Borexino Collaboration), Phys. Rev. D 82 (2010) 033006

[6] G. Bellini at al. (Borexino Collaboration), Phys. Rev. Lett. 108 (2012) 051302

[7] G. Bellini at al. (Borexino Collaboration), Phys. Rev. D 89 (2014) 112007

[8] G. Bellini at al. (Borexino Collaboration), Nature 512 (2014) 383-386

[9] G. Fiorentini, and B. Ricci, Comm. Mod. Phys. E1 (1999) 49, INFNFE-01-97

[10] G.A. Cowan, and W.C. Haxton, Los Alamos Science 3 (2) (1982) 46

[11] M. Agostini et al. (Borexino Collaboration), Phys. Rev. D 92 (2015) 031101

[12] M. Coltorti et al., Earth Planet. Sci. Lett. 293, 259 (2010)

[13] Y. Huang et al., Geochem., Geophy., Geosyst. 14 (2003) 2003

[14] G. Bellini et al. (Borexino Collaboration), Phys. Rev. C 81 (2010) 3

[15] G. Bellini et al. (Borexino Collaboration), Phys. Rev. D 85 (2012) 092003

[16] M. Agostini et al. (Borexino Collaboration), submitted to Phys. Rev. Lett.

[17] G. Bellini et al. (Borexino Collaboration), J. High Energy Phys. 08 (2013) 038 